Service social

\title{
L'évolution des services de santé et des services sociaux. La réaction des groupes d'aînés québécois
}

\section{Andrée Sévigny et Yves Hurtubise}

Volume 46, numéro 1, 1997

Politique et intervention

URI : https://id.erudit.org/iderudit/706752ar

DOI : https://doi.org/10.7202/706752ar

Aller au sommaire du numéro

Éditeur(s)

École de service social de l'Université Laval

ISSN

1708-1734 (numérique)

Découvrir la revue

Citer cet article

Sévigny, A. \& Hurtubise, Y. (1997). L'évolution des services de santé et des services sociaux. La réaction des groupes d'aînés québécois. Service social, 46(1), 119-146. https://doi.org/10.7202/706752ar
Résumé de l'article

Le débat entourant les politiques sociales québécoises est actuellement habité par trois grandes tendances : la tendance socioétatique, la tendance néolibérale et celle de la sociocommunautarisation. Cet article présente une analyse de contenu de documents publiés par les groupes d'aînés québécois (entre 1979 et 1995) et retrace la réaction de ces groupes en ce qui a trait à révolution des politiques québécoises de santé et de services sociaux, notamment en matière de soutien à domicile. Cette analyse renvoie à la conception du rôle de l'État et des services publics, aux raisons et aux valeurs qui sous-tendent les prises de position, de même qu'à la vision de la démocratie et de la place des aînés dans la société. Elle vise à déterminer dans laquelle des grandes tendances théoriques qui cohabitent au Québec les groupes d'aînés se situent. 


\title{
L'évolution des services de santé et des services sociaux La réaction des groupes d'aînés québécois
}

\author{
Andrée SÉVIGNY \\ Yves HURTUBISE
}

Le débat entourant les politiques sociales québécoises est actuellement habité par trois grandes tendances: la tendance socioétatique, la tendance néolibérale et celle de la sociocommunautarisation. Cet article présente une analyse de contenu de documents publiés par les groupes d'aînés québécois (entre 1979 et $1995)$ et retrace la réaction de ces groupes en ce qui a trait à l'évolution des politiques québécoises de santé et de services sociaux, notamment en matière de soutien à domicile. Cette analyse renvoie à la conception du rôle de l'État et des services publics, aux raisons et aux valeurs qui sous-tendent les prises de position, de même qu'à la vision de la démocratie et de la place des aînés dans la société. Elle vise à déterminer dans laquelle des grandes tendances théoriques qui cohabitent au Québec les groupes d'aînés se situent.

The debate surrounding Quebec's social policies is influenced at the present time by three major trends: the providentialism, the neoliberalism and the communautarism. This article presents an analysis of documents published by groups of elders from the province of Québec (between 1979 and 1995) and retraces the positions of these groups concerning the evolution of health and social policies in Québec, specially concerning home care services. This analysis refers to their perception of the state and of public services, to the rationale and the values supporting their position, their notion of democracy and of the place of elders in society. The aim of the analysis is to determine the theoretical trends that inspire these groups. 
Au Québec, comme ailleurs en Occident, nous assistons à ce qu'il est convenu d'appeler la crise de l'État-providence. Cette crise remet en cause l'ensemble des rapports entre l'État et la société civile, comme le notait Rosanvallon dès 1981. Dans un effort de sortie de crise, diverses tendances se dessinent en ce qui a trait au remaniement des politiques sociales. Actuellement, au Québec, trois principales tendances cohabitent : la tendance socioétatique, qui consiste à défendre la pertinence de garder les acquis de l'Etat-providence, la tendance néolibérale, qui vise à reconsidérer les politiques sociales dans la perspective d'une intervention minimale de l'État, et la tendance sociocommunautaire, qui propose la reconnaissance d'une autonomie accrue des personnes et des groupes communautaires et l'établissement d'un nouveau partenariat entre le secteur public et le secteur volontaire et communautaire (Favreau et Hurtubise, 1993; Lamoureux, 1994; Vaillancourt et Jetté, 1997). Ces grandes tendances sont aussi présentes dans les débats actuels entourant le soutien à domicile des personnes âgées.

Au Québec, surtout depuis les années 1970, les personnes âgées s'organisent, se regroupent et tissent des liens de solidarité à travers leurs disparités culturelles et idéologiques. Des aînés de notre société, regroupés, passent à l'action et s'expriment publiquement, afin de faire valoir leur opinion sur des décisions politiques les concernant. Par exemple, on a vu au printemps 1996 ces regroupements manifester leur désaccord sur certaines mesures prévues dans le nouveau régime d'assurance-médicaments du Québec. Ils ont eu l'occasion, à maintes reprises, de formuler leurs attentes, leurs positions idéologiques ou leurs recommandations à l'adresse du gouvernement québécois. Trois regroupements sont davantage présents sur la scène publique. La Fédération de l'âge d'or (FADOQ) a vu le jour en 1970, l'Association québécoise de défense des droits des personnes retraitées et préretraitées (AQDR) a été formée officiellement en 1979, alors que la Coalition des aînés du Québec (comptant 18 associations ou fédérations, dont la FADOQ et l'AQDR) a été créée en 1984.

Le présent article apporte un éclairage sur la position des organisations qui regroupent des personnes âgées (de façon locale, régionale ou nationale), au regard des décisions prises dans le domaine de la santé et des services sociaux, depuis la fin des années 1970, particulièrement dans le champ du soutien 
à domicile. En première partie de l'article, nous présentons succinctement les trois principales tendances qui cohabitent actuellement dans le débat public québécois sur le rapport Étatsociété. Dans un deuxième temps, nous présentons sommairement la méthode que nous avons utilisée pour rendre compte des positions officielles des personnes âgées regroupées; ces prises de position sont synthétisées et leur évolution dans le temps est soulignée. En troisième lieu, nous cherchons à déterminer dans laquelle de ces tendances les organisations québécoises de personnes âgées s'inscrivent. Le but de cet article est d'alimenter la réflexion de différents acteurs, notamment ceux du soutien à domicile, sur des voies de solution qui respecteront les personnes âgées et tiendront compte de leur vision. De plus, dans une perspective de l'analyse des mouvements sociaux, il apparaît intéressant de savoir dans quelle direction les différents regroupements de personnes âgées ont fait porter leur discours. En ce sens, dévoiler le discours des aînés permettra à la société - mais aussi à ces regroupements - de mieux articuler leur vision et de mieux se positionner dans le débat entourant les politiques sociales, particulièrement en ce qui a trait au soutien à domicile.

\section{TROIS TENDANCES SUR LES RAPPORTS ÉTAT ET SOCIÉTÉ}

Afin de caractériser chacune des trois principales tendances, nous avons retenu trois dimensions du discours des regroupements de personnes âgées : la conception qu'ils ont de l'État et des services publics, les raisonnements et les valeurs qui soustendent ces différentes tendances, de même que l'idée que l'on se fait de la démocratie et de la place des personnes bénéficiaires de services.

\section{La tendance socioétatique (État-providence)}

Dans le débat actuel sur les politiques sociales en matière de services de santé et de services sociaux, notamment de soutien à domicile, la tendance socioétatique est celle qui préconise l'intervention de l'État comme régulateur des rapports sociaux. Cette conception institutionnelle définit l'État comme le mécanisme de base pour assurer la protection sociale (Groulx, 1990). L'État a alors une fonction de fournisseur de services universels et gratuits, tandis que la famille et le marché n'ont qu'un rôle résiduel à jouer (Favreau et Hurtubise, 1993; Groulx, 1990; Rosanvallon, 1995). Pour Rosanvallon (1995, p. 93) «le principe 
universaliste a été historiquement au cœur de la conception civique et nationale de la Sécurité sociale », selon un plan qui " couvre l'ensemble de la population du pays contre l'ensemble des facteurs d'insécurité ». Les politiques sociales prennent donc la forme d'une institutionnalisation des besoins sociaux. Elles sont alors universelles et gérées par la sphère publique (Beauchemin, Bourque et Duchastel, 1995). Selon Groulx (1990) l'universalité renforcerait le sentiment d'appartenance collective, favoriserait l'intégration sociale des citoyens et viserait à combattre les inégalités sociales. Cette tendance repose aussi sur la croyance que les inégalités sociales sont inscrites dans les structures mêmes de la société (MSSS, 1988) et qu'elles sont produites par l'économie de marché. On reconnaît que l'origine des problèmes sociaux est plus sociale qu'individuelle et qu'il est nécessaire de remédier à ces problèmes collectivement (Mayer, 1994). Les providentialistes considèrent l'égalité comme un principe réparateur de l'injustice voulant que des individus soient désavantagés du fait de leur origine sociale (Beauchemin et collab., 1995). L'intervention de l'État dans les différentes sphères de la vie sociale est alors interprétée comme la réalisation de l'idée de démocratie. De plus, l'État-providence est porteur d'une notion de démocratie représentative qui s'appuie principalement sur une coalition majoritaire d'électeurs ayant intérêt à le maintenir (ex.: les femmes au travail, les personnes âgées, les employés de l'État) (Groulx, 1990).

\section{La tendance néolibérale}

Le courant néolibéral se compose à la fois de conservatisme politique et de libéralisme économique (Beaudry et Jalbert, 1987). Contrairement aux providentialistes, les néolibéraux ont une conception résiduelle du rôle de l'État dans le champ du social. Selon eux, la protection des risques doit être assurée d'abord par le marché et par la famille, qui sont les premiers responsables de la protection contre les risques sociaux (Groulx, 1990). Le néolibéralisme vise l'État minimum qui ne prévoit qu'un filet de sécurité sélectif pour protéger temporairement certains individus contre des aléas dont ils peuvent être victimes (Beaudry et Jalbert, 1987; Groulx, 1990; Sorman, 1984). D'après Sorman (1984), tenant du néolibéralisme, il importe de renouer avec la croissance grâce à la liberté d'entreprendre. Les valeurs essentielles du néolibéralisme sont le développement de l'autonomie et 
de l'initiative individuelle ainsi que la restauration du sens de l'économie et du sacrifice (Beauchemin et collab., 1995). Suivant ce raisonnement, l'individu devra de plus en plus assumer luimême les conséquences des risques sociaux. Les néolibéraux lient la démocratie au développement de l'individualisme et à l'efficacité économique (Sorman, 1984). Selon eux, la privatisation est une occasion de réconcilier la démocratie et l'efficacité économique tant recherchée. Sorman (1984) en appelle aux solutions alternatives en concurrence avec la sécurité sociale, afin de protéger les individus contre le despotisme bureaucratique et étatique. Les bénéficiaires de services sont alors vus comme des consommateurs. Les problèmes sociaux sont découpés de façon que des mesures adaptées à chaque situation particulière soient destinées à des groupes cibles. Beauchemin et collab. (1995) donnent l'exemple du gouvernement Mulroney qui a remplacé certaines politiques sociales universelles par des crédits d'impôt s'adressant à des groupes cibles (ex. : crédit d'impôt pour enfants, crédit d'impôt à la consommation). Selon ces auteurs, la réforme des politiques sociales du gouvernement libéral fédéral (projet Axworthy) est un autre exemple de cette vision néolibérale du bénéficiaire de services.

\section{La tendance sociocommunautaire}

La troisième voie, enfin, tente de redéfinir les rapports entre l'État et la société civile (Rosanvallon, 1981). Rosanvallon (1981) invite les acteurs sociaux à sortir de l'alternative privatisationétatisation et à redonner de la vigueur à la solidarité. Pour les tenants de la voie sociocommunautaire, c'est la communauté qui constitue le lieu le plus proche de la source des problèmes et qui permet d'accroître la coopération, l'entraide et la responsabilisation devant les problèmes (Groulx, 1990). Ils reconnaissent le rôle essentiel de l'État pour maintenir les solidarités et la cohésion sociale et réaffirment le caractère universel que doivent garder les services publics. Cependant, il leur importe de repenser l'État-providence pour qu'il puisse continuer à jouer son rôle (Rosanvallon, 1981, 1995). La voie sociocommunautaire reposerait, selon Groulx (1990), sur des valeurs de solidarité et de participation populaire. La responsabilité des services doit être remise à des instances proches des citoyens utilisateurs (Groulx, 1990; Rosanvallon, 1981). Cette nouvelle dynamique implique la reconnaissance d'acteurs sociaux ayant une histoire, des valeurs, des façons de faire différentes: État, bénéficiaires des 
services, réseaux primaires, groupes communautaires. Selon les défenseurs de la voie sociocommunautaire, il est possible de construire des formes de partenariat entre l'institutionnel et le communautaire dans l'offre de services (Plamondon, 1991). La notion de partenariat ferait appel à un rapport social qui mise sur l'échange et la négociation plutôt que sur l'affrontement, même si l'un n'exclut pas l'autre. Cette volonté de faire une plus grande place à la société civile repose sur la conviction, formulée par Rosanvallon (1981, p. 122), que la société ne fonctionne pas selon un schéma individualiste. II rappelle la réflexion de Pitrou qui souligne l'importance économique des réseaux souterrains de solidarité familiale. Dans le champ du soutien à domicile québécois, plusieurs auteurs confirment cette thèse lorsqu'ils affirment que la famille est déjà très engagée dans les stratégies de soutien à domicile des aînés (Garant et Bolduc, 1990; Guberman et Maheu, 1994). Sans les proches et la communauté, le soutien à domicile serait impossible. La voie sociocommunautaire s'appuie sur la notion d'équité : "égalité dans la différence » (Groulx, 1990, p. 109). L'équité devient une action compensatoire pour les inégalités sociales. En contre-partie, les membres de la société doivent aussi s'impliquer (Rosanvallon, 1995). La sociocommunautarisation vise la démocratie de la participation directe (Groulx, 1990). Dans cette optique, les organismes communautaires sont vus comme des lieux d'expérimentation pour apprendre à "faire autrement", des espaces sociaux où les gens sont appelés à se réapproprier des moyens d'agir sur leur vie et ainsi conserver un peu de pouvoir sur leurs conditions de vie (Plamondon, 1991). II importe de permettre aux individus de retrouver leur capacité de changer et de régler leurs problèmes de façon autonome (Lamoureux, 1994). Ainsi, le bénéficiaire de services est considéré comme un coproducteur de ces services. II est un acteur, responsable de son devenir, mais la société a l'obligation de lui fournir les moyens pour le réaliser (Rosanvallon, 1995). C'est ce que Godbout (1995) appelle la troisième génération de l'État-providence.

\section{LA MÉTHODOLOGIE}

Pour la présente recherche, 38 documents ont été répertoriés à la Bibliothèque nationale et auprès des groupes eux-mêmes. Les index du Journal des débats ont été consultés de 1966 (année de la création de la commission Castonguay-Nepveu) jusqu'à 
l'automne 1995. Nous avons ainsi pu établir la liste des documents présentés par les groupes de personnes aînées à des commissions parlementaires ou à des commissions d'enquête. Ces documents présentent donc la position officielle de ces groupes et révèlent ainsi leurs orientations politiques et idéologiques. L'analyse qualitative du contenu de ces documents est à la source du présent article. Elle renvoie à vingt-sept de ces documents (voir le tableau 1). Les onze autres documents ne comportent aucun élément lié aux thèmes à l'étude. De plus, aucun mémoire n'a été présenté par des groupes d'aînés lors de la commission d'enquête sur la santé et le bien-être social à la fin des années 1960 (Castonguay-Nepveu). Le premier document répertorié au moment de la collecte des données a été réalisé par l'AQDR en 1979. II importe de préciser que seize des vingtsept documents retenus pour l'analyse sont produits par les instances provinciales des groupes, alors que les onze autres proviennent de sections régionales ou de groupes locaux d'aînés.

Aux fins de l'analyse, le thème est considéré comme unité d'enregistrement (Bardin, 1986). Les thèmes correspondent aux trois dimensions retenues pour l'étude : la conception de l'État et des services publics, la conception de la démocratie et de la place des personnes bénéficiaires de services, de même que les raisons et les valeurs qui sous-tendent ces conceptions. Tous les mémoires recensés ont été lus à plusieurs reprises, afin de procéder à un repérage systématique des extraits significatifs pour l'objectif analytique (Bardin, 1986, p. 105). Le matériel recueilli sous chaque thème a ensuite fait l'objet d'une subdivision plus fine. Cette seconde étape de découpage du matériel a permis de dégager, a posteriori, un certain nombre de souscatégories qui nous ont servi à présenter et à interpréter les positions des groupes de personnes aînées. 


\section{Tableau 1}

\section{Liste des documents analysés classés selon l'année de parution}

\begin{tabular}{|c|c|c|}
\hline Année & Organisme & Repère bibliographique ou historique \\
\hline 1979 & AQDR & Manifeste Vieillir chez soi \\
\hline 1980 & FADOQ & Mémoire présenté au ministre des Affaires sociales \\
\hline 1986 & AQDR (Saint-Félicien) & $\begin{array}{l}\text { Mémoire présenté à la commission Rochon (vol. } 21 \text {, } \\
\left.n^{0} 10382\right)^{*}\end{array}$ \\
\hline $1986 a$ & AQDR (Saguenay) & $\begin{array}{l}\text { Mémoire présenté à la commission Rochon (vol. } 21 \text {, } \\
n^{\circ} \text { 10383) }\end{array}$ \\
\hline $1986 b$ & AQDR (Saguenay) & $\begin{array}{l}\text { Mémoire présenté à la commission Rochon (vol. } 21 \text {, } \\
n^{0} 10383-01 \text { ) }\end{array}$ \\
\hline 1986 & $\begin{array}{l}\text { AQDR (Longueuil et } \\
\text { Sorel) }\end{array}$ & $\begin{array}{l}\text { Mémoire présenté à la commission Rochon (vol. } 28 \text {, } \\
\left.n^{0} 10515\right)\end{array}$ \\
\hline 1986 & AQDR (Charlesbourg) & $\begin{array}{l}\text { Mémoire présenté à la commission Rochon (vol. } 27 \text {, } \\
n^{0} \text { 10493) }\end{array}$ \\
\hline $1986 a$ & FADOQ & $\begin{array}{l}\text { Mémoire présenté à la commission Rochon (vol. } 10 \text {, } \\
n^{0} 10162 \text { ) }\end{array}$ \\
\hline $1986 b$ & FADOQ & $\begin{array}{l}\text { Mémoire présenté à la commission Rochon (vol. 10, } \\
n^{0} 10162-01 \text { ) }\end{array}$ \\
\hline 1986 & $\begin{array}{l}\text { Forum des citoyens } \\
\text { âgés de Montréal }\end{array}$ & $\begin{array}{l}\text { Mémoire présenté à la commission Rochon (vol. } 2 \text {, } \\
n^{0} 10024 \text { ) }\end{array}$ \\
\hline 1986 & $\begin{array}{l}\text { Golden Age Associa- } \\
\text { tion }\end{array}$ & $\begin{array}{l}\text { Mémoire présenté à la commission Rochon (vol. } 40 \text {, } \\
n^{\circ} 10756,756-1,2,3,4 \text { ) }\end{array}$ \\
\hline $1986 a$ & $\begin{array}{l}\text { NDG (Notre-Dame- } \\
\text { de-Grâce) Senior } \\
\text { Citizens' Council }\end{array}$ & $\begin{array}{l}\text { Report of the task force on foster care of the elderly in } \\
\text { NDG. Mémoire présenté à la commission Rochon } \\
\left(n^{\circ} 10065\right)\end{array}$ \\
\hline $1986 b$ & $\begin{array}{l}\text { NDG (Notre-Dame- } \\
\text { de-Grâce) Senior } \\
\text { Citizen's council }\end{array}$ & $\begin{array}{l}\text { Mémoire présenté à la commission Rochon }\left(n^{0} 10065 \text { - }\right. \\
01)\end{array}$ \\
\hline 1986 & $\begin{array}{l}\text { Seniors of Westmount } \\
\text { Action Group }\end{array}$ & $\begin{array}{l}\text { Mémoire présenté à la commission Rochon (vol. } 33 \text {, } \\
n^{0} 10618 \text { ) }\end{array}$ \\
\hline 1988 & AQDR & $\begin{array}{l}\text { Mémoire présenté à la Commission des affaires } \\
\text { sociales (sécurité du revenu) }\end{array}$ \\
\hline $1990 a$ & FADOQ & $\begin{array}{l}\text { Améliorer la qualité de vie des aînés. Mémoire présenté } \\
\text { au Gouvernement du Québec }\end{array}$ \\
\hline $1990 b$ & FADOQ & $\begin{array}{l}\text { Améliorer la qualité de vie des aînés. Mémoire présenté } \\
\text { au caucus québécois du Parti progressiste-conservateur } \\
\text { du Canada }\end{array}$ \\
\hline
\end{tabular}


TABleau 1 (suite)

\begin{tabular}{|c|c|c|}
\hline Année & Organisme & Repère bibliographique ou historique \\
\hline $1990 a$ & $\begin{array}{l}\text { Coalition des aînés du } \\
\text { Québec }\end{array}$ & $\begin{array}{l}\text { Déclaration de principes sur la santé et les services } \\
\text { sociaux }\end{array}$ \\
\hline $1990 b$ & $\begin{array}{l}\text { Coalition des aînés du } \\
\text { Québec }\end{array}$ & $\begin{array}{l}\text { Mémoire présenté à la Commission des affaires so- } \\
\text { ciales (Commission sur l'avant-projet de loi sur les ser- } \\
\text { vices de santé et les services sociaux, tome Xil, 163M) }\end{array}$ \\
\hline 1991 & FADOQ & $\begin{array}{l}\text { Commentaires sur la réforme de la santé et des ser- } \\
\text { vices sociaux }\end{array}$ \\
\hline 1992 & FADOQ & $\begin{array}{l}\text { Les } 50 \text { ans et plus, la santé et le bien-être : un espace à } \\
\text { construire }\end{array}$ \\
\hline 1992 & n des aînés du & $\begin{array}{l}\text { Point de vue des aînés du Québec sur le document Un } \\
\text { financement à la mesure de nos moyens, présenté à la } \\
\text { Commission des affaires sociales (Commission sur } \\
\text { l'avant-projet de loi sur les services de santé et les } \\
\text { services sociaux, tome } 1,2 \mathrm{M} \text { ) }\end{array}$ \\
\hline 1993 & $\begin{array}{l}\text { Coalition des aînés du } \\
\text { Québec }\end{array}$ & Manifeste pour les élections fédérales de 1993 \\
\hline 1994 & AQDR & $\begin{array}{l}\text { La régionalisation en matière de santé et de services } \\
\text { sociaux }\end{array}$ \\
\hline 1994 & $\begin{array}{l}\text { Coalition des aînés du } \\
\text { Québec }\end{array}$ & $\begin{array}{l}\text { Énoncé des préoccupations des aînés présenté au Gou- } \\
\text { vernement du Québec }\end{array}$ \\
\hline 1995 & $\begin{array}{l}\text { Coalition des aînés du } \\
\text { Québec }\end{array}$ & $\begin{array}{l}\text { Mémoire présenté à la Régie régionale de la santé et des } \\
\text { services sociaux (Montréal-Centre) }\end{array}$ \\
\hline 1995 & $\begin{array}{l}\text { NDG (Notre-Dame- } \\
\text { de-Grâce) Senior } \\
\text { Citizens' Council }\end{array}$ & $\begin{array}{l}\text { Brief to la Régie régionale de la santé et des services } \\
\text { sociaux Montréal-Centre (concernant le virage ambula- } \\
\text { toire) }\end{array}$ \\
\hline
\end{tabular}

* Les notes entre parenthèses sont des repères bibliographiques pour retrouver le document à la Bibliothèque nationale du Québec.

\section{L'ANALYSE DE LA VISION DES AÎNÉS REGROUPÉS}

Comment les regroupements québécois de personnes âgées se sont-ils situés dans ce débat concernant l'avenir des politiques sociales? Nous avons identifié, dans les documents consultés, des prises de position, notamment sur le rôle de l'État, des groupes communautaires et du secteur privé lucratif ainsi que sur les services eux-mêmes. Dans un deuxième temps, nous nous sommes attardés aux valeurs ainsi qu'aux raisons qui soustendent ces prises de position. En troisième lieu, nous avons porté une attention particulière à la vision que ces organisations ont de la démocratie et de la place des aînés dans les instances décisionnelles. 


\section{La position des groupes de personnes âgées à l'égard des services}

\section{Le rôle de l'État et le panier de services}

D'entrée de jeu, il est possible de constater que, selon les aînés, l'État doit jouer un rôle central dans la réponse à leurs divers besoins. En 1979 l'AQDR déclare à cet effet :

Ce qui est cependant de plus en plus clair, c'est que les personnes âgées ont des besoins, qu'ils sont pressants et que l'État devra y répondre et affecter les ressources humaines et financières qui permettront de les satisfaire (p. 22).

Cette position se confirme dans le temps. Les groupes d'aînés désirent garder les droits acquis durant la période où s'est développé l'État-providence. Par exemple, la Coalition des aînés (1992) s'oppose à toute atteinte à l'universalité des pensions de vieillesse. La section de l'AQDR de Longueuil et Sorel en 1986, la FADOQ en 1991 et la Coalition des aînés en 1993 se prononcent contre l'imposition d'un ticket modérateur. Selon l'AQDR (AQDR Longueuil et Sorel, 1986), l'imposition de frais modérateurs et la privatisation sont des opérations qui visent à saboter le système public. Elle souhaite une réorganisation des budgets, mais pas le retrait de l'État. En 1992, la FADOQ est aussi d'avis que les préoccupations des gouvernements sont strictement budgétaires et elle les invite à élaborer une vision plus globale de la situation. Cette même année, la Coalition des aînés (1992) s'oppose fermement à toute décision de l'État visant à réduire le panier des services assurés en vue d'introduire une tarification sur certains services de base. En matière de soutien à domicile, dès 1979, l'AQDR souhaite que le gouvernement prenne les mesures nécessaires pour que tous les CLSC existants (et, dans les régions où il n'en existe pas, les organismes compétents) soient munis de services et de budgets adéquats pour la mise en place de programmes complets. L'AQDR (1979) souhaite aussi qu'il y ait une répartition plus adéquate des soins et des services à domicile dans les régions urbaines et dans les régions éloignées. Cette volonté de privilégier le développement des services provenant des CLSC se confirme dans le temps et chez plusieurs groupes d'aînés tant du milieu francophone que du milieu anglophone. En 1990, c'est la FADOQ (1990a) qui revient à la charge et recommande au ministère de la Santé 
et des Services sociaux de munir les CLSC des ressources humaines et financières suffisantes pour rendre leur programme de soutien à domicile efficace à la grandeur du territoire québécois.

\section{Le rôle de l'État et des groupes communautaires}

Par ailleurs, les aînés adressent certaines critiques à l'appareil étatique. Selon eux, la rigidité, la bureaucratie et le contrôle directif inhérents aux services étatiques ne permettent pas l'exercice d'un bon jugement dans l'application des services et entraînent des retards dans la réalisation des plans de services (AQDR, 1979; Forum, 1986). La multiplicité des structures et le morcellement des services ne favorisent pas l'intégration des individus, jeunes ou moins jeunes, dans un réseau à grandeur humaine (FADOQ, 1986a). Depuis 1979, les aînés signalent l'insuffisance de services publics, notamment en matière de soutien à domicile. À partir de 1986 le discours de plusieurs groupes dénonce aussi le phénomène de l'alourdissement des clientèles et la longueur des listes d'attente comme des situations problématiques. Ces groupes souhaitent la mise en place de nouveaux services (ex. : grand ménage saisonnier, entretien extérieur, transport, centres de jour et centres communautaires). D'un même souffle, ils souhaitent l'implantation des services bénévoles en complémentarité avec les services du réseau public. Ils insistent sur la vocation préventive des réseaux d'entraide. Selon certains groupes, l'Etat devrait soutenir et financer ces organismes bénévoles. La FADOQ considère que les organismes bénévoles «fournissent le complément d'aide [...] qui fait la différence entre un soutien à domicile efficace et un soutien à domicile à la fois efficace et enveloppant » (FADOQ, 1990a, p. 9). Cependant, la FADOQ (1990a, p. 9) et la Coalition des aînés (1990b) affirment en même temps qu'il ne faudrait pas traiter les organismes bénévoles comme du cheap labor. Déjà en 1986, l'AQDR de Longueuil et Sorel avait dénoncé la mobilisation trop forte des ressources bénévoles « en regard de leur gratuité » (1986, p. 3). La FADOQ et la Coalition suggèrent d'y intégrer les personnes âgées à tous les niveaux décisionnels. D'ailleurs, en ce qui a trait à l'implantation d'organismes bénévoles, les aînés eux-mêmes sont très actifs. La plupart de ces groupes incluent la défense des droits dans leurs activités, qui vont des services directs d'aide à domicile au transport, à l'information, à l'éducation, aux visites d'amitié ou aux loisirs. Ils estiment que ces services touchent plusieurs aspects de la vie 
des aînés qui ne sont pas couverts par les services publics. Ils affirment qu'une telle organisation ne peut être possible à l'intérieur du réseau public, professionnel et bureaucrate. Ils désirent être reconnus par la loi et appuyés financièrement par l'État, insistant sur le fait que ce type de services coûte moins cher que les services étatiques. Ils considèrent qu'il existe une division trop rigide entre les organismes créés par l'État et ceux qui ne le sont pas. En ce sens, la Coalition des aînés affirme que :

Ces organismes [communautaires] sont responsables devant les usagers locaux et ont la confiance de ces derniers. Ils méritent d'être soutenus comme membres précieux du réseau de la prévention, tant dans l'orientation des politiques que dans la fourniture de services (1990a, p. 2).

De son côté, la FADOQ considère que :

À travers leur action, ils [les organismes communautaires] donnent à un large éventail d'individus une voix et des moyens de vivre mieux [...] Derrière cette action, des retombées sociales de taille passent souvent inaperçues. En retrouvant leur dignité, en expérimentant la justice, en sortant de l'isolement, en pratiquant des réseaux de solidarité, une foule de gens retrouvent le goût de vivre et de participer au lieu de se retirer [...] À ce titre, ils contredisent directement la tendance au néolibéralisme sauvage et reposent [sic] constamment l'importance des solidarités (1992, p. 56).

Cependant, depuis le début des années 1990 surtout, certains groupes d'aînés s'inquiètent quant à l'emprise de l'État sur les groupes communautaires. En réaction à l'avant-projet de loi sur la santé et les services sociaux (loi 120), la Coalition estime qu'il vaudrait mieux pour la population qu'on conserve aux organismes communautaires l'originalité de leur démarche au lieu de les enfermer dans des programmes de services qui réduiront une action sociale s'attaquant aux causes mêmes du problème. Les membres de la Coalition estiment que le gouvernement cherche à assimiler le réseau des organismes bénévoles et ses membres dans le but de compléter les services du réseau public et porte ainsi atteinte à "l'autonomie des organismes communautaires, à leur dynamisme et à leur potentiel innovateur » (Coalition, 1990b, p. 9). Selon la FADOQ (1992), les organismes 
communautaires doivent jouer un rôle complémentaire à celui du réseau public, et non un rôle substitutif.

\section{Le rôle du secteur privé lucratif}

En ce qui concerne la place du secteur privé à but lucratif, l'AQDR se prononce très clairement contre cette solution. Déjà en 1979, l'AQDR dénonçait les abus du privé dans les foyers et les résidences pour personnes âgées. En 1986, la section de Saint-Félicien de l'AQDR s'est prononcée contre les allocations directes (allocation financière versée directement au bénéficiaire afin qu'il engage une ressource). Elle allègue que cette formule crée beaucoup de confusion à savoir qui est l'employeur et qu'elle frise l'illégalité tout en encourageant le travail au noir des femmes. Ce sont effectivement les femmes qui sont surtout engagées pour réaliser les tâches d'aide à domicile. En 1992, la Coalition des aînés émet aussi des réticences à l'égard de cette mesure et souhaite qu'une telle mesure soit supervisée attentivement. En 1986, lors des audiences de la commission Rochon, la section du Saguenay de l'AQDR (1986b) dénonce le retrait de l'État et la part de plus en plus grande faite aux ressources du secteur privé à but lucratif.

De son côté, la FADOQ (1990b, p. 5) laisse une place aux initiatives du privé. À cet effet, elle déclare : « [...] il faut soutenir les organismes publics et privés qui facilitent le maintien à domicile et favorisent ainsi l'autonomie des personnes âgées ». Les regroupements d'aînés anglophones de Montréal manifestent moins de réticences à l'égard de la mise en place de services privés. La Golden Age Association (1986, p. 2) recommande à la commission Rochon de "reconnaître le besoin d'allouer des fonds aux organismes privés et bénévoles dont les programmes et les services aident à maintenir l'auto-estime, l'autonomie et l'utilité des aînés au sein de la communauté ". Dans un document portant sur les familles d'accueil pour aînés, présenté à la commission Rochon, le NDG Senior Citizens' Council (1986a) affirme que le privé ne doit pas être ignoré. Cependant, il recommande que des normes soient clarifiées pour éviter, entre autres choses, que se créent des services différents pour les riches et pour les pauvres. En 1996, en commission parlementaire, la Coalition des aînés du Québec réclame que l'Etat reste maître de la gestion du régime d'assurance-médicaments et dénonce la trop grande place faite aux assureurs privés (Presse canadienne, 1996). 


\section{Les programmes d'employabilité}

En ce qui concerne les programmes d'employabilité, les aînés en appellent à la permanence des services, mais aussi à la compétence des personnes-ressources. En 1986, l'AQDR du Saguenay (1986a) souhaitait déjà que les employés des programmes d'employabilité soient formés pour l'approche aux aînés. En 1988, l'instance provinciale de l'AQDR s'inquiète non seulement du sort des bénéficiaires âgés, mais aussi du sort des participants à ces programmes d'emploi :

Tous les efforts concernant la mise en place et l'application de programmes de formation ou de développement de l'employabilité ne prendront vraiment un sens que dans la mesure où les usagers de ces programmes peuvent espérer un emploi stable, satisfaisant et rémunérateur (AQDR, 1988, p. 3).

En 1995, la position de certains groupes est très ferme: la Coalition des aînés et le NDG Senior Citizens' Council expriment clairement leur opposition à l'utilisation des prestataires de la sécurité du revenu, par l'intermédiaire de divers programmes, afin de fournir des services de soutien à domicile ou d'aide au foyer. Selon eux, rien ne garantit la qualité, la continuité des services et la sécurité dont les personnes âgées ont besoin. Par contre, en 1990, la FADOQ (1990a, p. 8) affirme qu'il faut que « [...] à tout prix on empêche le travail au noir d'envahir ce domaine ». La FADOQ (1990b, p. 6) considère que les programmes d'employabilité peuvent contribuer à ramener les " sans-travail » sur le marché de l'emploi. De son côté, le Seniors of Westmount Action Group proposait, en 1986, que des emplois soient créés pour des personnes qui veulent retourner sur le marché du travail et qui peuvent accomplir des tâches d'aide à domicile. Ce regroupement recommandait alors la fondation d'organismes à but non lucratif qui apporteraient des services complémentaires à ceux offerts par les employés des CLSC. Malgré certaines divergences d'opinions, tous ces groupes souhaitent des programmes qui créent des emplois décents et qui offrent la stabilité des services aux bénéficiaires.

\section{La qualité des services}

En ce qui a trait à la qualité des services, les groupes d'aînés désirent que les personnes ayant besoin de ces services soient 
traitées comme des personnes à qui on donnera des soins plus humains (Coalition des aînés, 1990a; FADOQ, 1980). La FADOQ insiste pour que, dans l'application de sa politique de soutien à domicile, le Ministère veille à ce que les médecins, les infirmières et infirmiers ainsi que les auxiliaires et les aides familiaux leur prodiguent des soins et des services attentifs qui répondent à leurs besoins et les satisfassent entièrement (1980).

\section{Le raisonnement et les valeurs qui sous-tendent cette vision}

\section{L'hébergement en institution et l'autonomie des personnes}

À travers le temps, les documents consultés témoignent du désir des aînés de demeurer à domicile le plus longtemps possible. Déjà en 1979, l'AQDR affirmait qu'au Québec on a trop misé sur l'hébergement en institution des aînés et pas assez sur la valorisation des ressources de l'individu et de son milieu de vie. Le soutien à domicile est vu comme un moyen pour éviter les dépenses astronomiques que nécessiteraient l'hébergement en institution ou l'engorgement des urgences. Toutefois, les aînés reconnaissent les limites de l'entraide familiale et communautaire. À cet effet, l'AQDR réaffirme en 1988 qu'un changement de mentalité dans notre société a éliminé le réseau d'appui et d'entraide dont la cellule familiale a besoin. Selon l'Association, la politique dans le domaine de la santé et des services sociaux va à contresens de cette réalité sociale lorsqu'elle propose que la famille assume davantage de responsabilités. De plus, les ressources bénévoles ne sont pas toujours disponibles et certains aînés préfèrent se priver de services de peur de déranger. La notion d'autonomie est centrale pour les aînés. Ils affirment depuis 1979 que toutes les personnes âgées ont droit aux services nécessaires au maintien de l'autonomie, au respect de leur dignité et au maintien du sentiment d'utilité. Certains groupes estiment que les services à domicile ne doivent pas être considérés comme une fin en soi, mais comme une aide au maintien de l'autonomie et à la transformation du milieu de vie (AQDR Saguenay, 1986b, Coalition des aînés, 1990b; FADOQ, 1990a). Ils dénoncent même la désinstitutionnalisation lorsqu'elle n'est que synonyme de coupures budgétaires (AQDR Longueuil et Sorel, 1986). À leur avis, il y a danger que la personne âgée soit gardée "entre ses quatre murs » (AQDR Longueuil et Sorel, 1986, p. 1) pour qu'elle coûte 
moins cher à l'ensemble des Québécois. Des ressources d'hébergement, des ressources intermédiaires et des services communautaires doivent être disponibles (FADOQ, 1986b; Coalition des aînés) pour éviter les situations où des personnes âgées sont maintenues à domicile sans les services nécessaires pour répondre à leurs besoins.

\section{L'universalité, l'accessibilité des services et la gratuité}

Les groupes d'aînés s'appuient sur les principes de gratuité, d'accessibilité et d'universalité des services. Selon eux, malgré l'importance d'assainir le système public, il faut conserver l'universalité des services, sinon il y aura création d'une " assurancemaladie des riches qui, elle, serait privée et une assurancemaladie des pauvres » (AQDR Longueuil et Sorel, 1986, p. 6). Pour la Coalition des aînés, l'universalité signifie :

[...] que les services doivent être disponibles à toute personne qui répond aux critères reconnus. Ceux-ci ne doivent pas établir de distinction basée sur la capacité de payer, la race, la langue ou le sexe. Elles peuvent, cependant, faire référence à des catégories d'âge, à la santé, ou à des besoins physiques ou sociaux, de sorte que toutes les personnes comprises dans ces groupes puissent se prévaloir des services (1990a, p. 1).

En 1990, la Coalition des aînés (1990b, p. 4) allègue, devant la Commission des affaires sociales, que les principes de gratuité et d'universalité sont "écorchés ". Selon elle, les longues listes d'attente pour l'obtention de services contredisent ce principe. De son côté, la FADOQ (1990a, p. 1) demande au gouvernement du Québec de faire le maximum d'efforts pour empêcher que les aînés à faibles et à moyens revenus « ne glissent progressivement dans une pauvreté humiliante ». Elle affirme :

[...] qu'une société se donne certains domaines où tous les citoyens ont le même traitement paraît un signe certain d'un désir de justice et d'égalité. Pareil choix de société empêche tout gestionnaire d'intervenir selon ses intérêts, ses goûts ou ses préférences pour décider qui paiera et combien, qui aura droit et pour combien de temps. [...] Les hommes politiques [...] doivent contrôler l'appétit des uns et protéger la faiblesse des autres (FADOQ, 1990a, p. 7). 
Les interventions des aînés s'appuient sur des principes d'égalité et de justice, particulièrement depuis les années 1990. Pour la Coalition des aînés (1990a, p. 1) l'égalité signifie « [...] le droit de chacun à recevoir des services équivalents en qualité et en quantité sans égard à l'âge, au sexe, à la classe sociale ou à l'origine ethnique ». Pour la Coalition (1990a, p. 3), " [...] les services privatisés mènent à l'inégalité, et souvent à des services de pauvre qualité, alors que les services bureaucratiques conduisent à un manque d'humanisme et à l'inflexibilité ". Les soins de santé doivent être accessibles à tous, sans distinction de classe (Coalition des aînés, 1994). De son côté, la FADOQ (1990b) insiste sur l'application de mesures qui allègent le fardeau financier des gens à faibles revenus et qui visent à assurer l'égalité financière aux femmes. La FADOQ (1992, p. 4) décrit le modèle proposé par Castonguay-Nepveu au début des années 1970 comme étant fondé sur les principes de solidarité collective et d'équité sociale qui devraient, selon elle, inspirer le gouvernement actuel; ce modèle était animé à la base par un souci d'équité sociale et une volonté " de répondre collectivement à des besoins collectifs » (FADOQ, 1992, p. 5). La Coalition des aînés (1992) abonde dans le même sens et affirme que l'État n'a pas prouvé que la crise budgétaire actuelle justifie un changement majeur de politique qui remettrait en cause des acquis comme l'universalité et la gratuité. La Coalition est d'avis qu'il faut non seulement éviter de hausser les taxes ou les charges supplémentaires, mais également maintenir le niveau de vie des aînés au-dessus du seuil de pauvreté.

\section{L'approche globale et intersectorielle}

Les divers groupes s'entendent pour défendre les vertus de la prévention et considèrent qu'il s'agit d'une logique à privilégier afin de préserver la santé physique et mentale des aînés et de diminuer les coûts liés aux interventions curatives et plus lourdes. Depuis la commission Rochon, des groupes (AQDR Saguenay, 1986b; FADOQ, 1986a, 1990a) dénoncent la large part laissée aux interventions curatives et ponctuelles comparativement aux activités préventives. L'attitude préventive est aussi liée à la notion d'autonomie: "l'attitude préventive nous apparaît nécessaire surtout si on veut stimuler le désir d'autonomie et pas seulement prodiguer des soins " (AQDR Longueuil et Sorel, 1986).

De plus, depuis 1979 , les aînés préconisent une approche globale et intersectorielle. Selon eux, les différents besoins des 
aînés sont étroitement reliés. L'intervention, notamment dans le champ du soutien à domicile, devrait tenir compte de toutes les dimensions de la personne. Les aînés dénoncent le manque de communication entre les services médicaux et les services sociaux du secteur public qui accentuerait même l'impuissance du citoyen forcé de demander des services et en même temps de faire la preuve qu'il en a réellement besoin (AQDR, 1979). Dès 1980 , la FADOQ proposait la formation d'un conseil interministériel afin d'éviter les déboires des interventions gouvernementales multiples et fragmentaires. En 1986, la FADOQ (1986b) en appelle à une complémentarité des services dans les centres de jour et les centres communautaires. Enfin, certains groupes remettent en question la primauté des interventions professionnelles (FADOQ, 1992; NDG Senior Citizens' Council, 1986b). Pour sa part, la Coalition des aînés (1990a) considère qu'une plus grande attention devrait être portée aux besoins des usagers des services et aux préférences qu'ils expriment avant d'imposer le point de vue des professionnels. À cet effet, le NDG Senior Citizens' Council (1986b) estime qu'un réseau public fondé, entre autres, sur la prestation de services par des professionnels ne peut pas permettre l'engagement volontaire et laisser la place aux aînés comme décideurs, comme c'est le cas dans les organismes communautaires.

\section{Une vision globale de la personne âgée}

Certains groupes d'aînés considèrent aussi que les politiques sociales les concernant doivent s'inscrire dans une vision plus globale de l'ensemble de la société. En 1980, la FADOQ suggérait l'élaboration d'une politique de la famille conçue et appliquée de façon que les aînés ne soient pas exclus de leur milieu naturel et que la société reconnaisse aussi le rôle qu'elle peut jouer à leur égard. En 1986, cette vision était partagée et défendue par la section de l'AQDR de Longueuil et Sorel (1986) qui affirmait l'importance pour tous les intervenants (publics et communautaires) de modeler leurs objectifs futurs sur une politique d'ensemble qui confirmera le statut des personnes âgées afin qu'elles puissent continuer de participer activement à la société. Certains groupes en appellent à un changement de mentalité à l'égard du vieillissement, afin d'éviter la dévalorisation et la démobilisation des personnes âgées (FADOQ, 1986a; AQDR Longueuil et Sorel, 1986). Changement de mentalité qui devrait être vécu aussi par 
les aînés eux-mêmes, afin qu'ils acceptent la venue des intervenants à domicile et favorisent les programmes d'échanges intergénérationnels visant l'intégration sociale de la personne âgée (AQDR Longueuil et Sorel, 1986).

\section{Leur vision de la démocratie et de la place des aînés}

L'analyse des écrits révèle la volonté des aînés d'être des partenaires de l'État et des organismes de services et de participer aux prises de décision les concernant. Ils estiment avoir contribué à l'évolution de la société et, de ce fait, avoir le droit de formuler leurs attentes. Ils dénoncent l'exclusion dont ils sont victimes. Selon eux, cette exclusion se manifeste par la réduction de leurs budgets, une santé fragile, une rareté de vie sociale, une dévalorisation par les autres membres de la société, une exclusion du monde du travail ou le manque de représentation directe. L'AQDR de Charlesbourg (1986) considère que les aînés doivent être représentés directement, sans l'intermédiaire de chercheurs, de techniciens de l'État ou de syndicalistes. L'AQDR de Charlesbourg (1986) affirme que les aînés, en plus de n'avoir aucun pouvoir de décision, n'ont aucun moyen d'expression et elle propose la tenue d'États généraux de la vieillesse. La FADOQ (1990a, 1991) et la Coalition des aînés (1990a) vont dans le même sens. Elles en appellent à la participation active des personnes âgées elles-mêmes. La FADOQ affirme que « les aînés sont des citoyens à part entière avec leurs caractéristiques propres et qu'ils sont désireux et parfaitement capables de participer à l'élaboration de toutes les décisions qui les concernent » (FADOQ, 1990a, p. 1). Les aînés s'expriment en faveur d'une décentralisation des décisions vers les instances régionales et locales qui favoriserait le développement de services plus près de leurs besoins tout en leur permettant de se positionner comme membres actifs dans les différentes instances décisionnelles. Déjà, en 1979, l'AQDR, qui réclamait des moyens de transport adaptés aux besoins des aînés, proposait que les différentes tables locales ou régionales de l'AQDR voient à l'application concrète des modalités de ce transport (prix horaire). En 1986, la section de l'AQDR de Longueuil et Sorel recommande qu'on leur réserve des places aux conseils d'admi-nistration des centres de jour ou des centres communautaires. La FADOQ (1986a, 1992) abonde dans le même sens et affirme l'importance que les aînés accordent à leur participation dans les collectivités locales et régionales au niveau des prises de décision. 
En 1994, l'AQDR juge que la régionalisation comporte certains avantages, comme :

[...] une plus grande adaptabilité du système, plus d'efficience, la nécessité d'une meilleure coordination et intégration locale/régionale, plus de motivation pour les professionnels et les professionnelles dans le but de mieux répondre aux besoins de la population et de favoriser la participation de la population au développement social et à la gestion des services sociosanitaires (1994, p. 1).

Cependant, la Coalition des aînés (1990b, p. 6) ne voit pas d'un bon œil la formation des régies régionales qui ne s'inscrirait pas dans une démarche de véritable régionalisation, mais qui serait plutôt une " déconcentration administrative ", ces dernières ne disposant pas d'un pouvoir réel d'orientation et de décision. La Coalition estime que :

La décentralisation ne doit pas s'entendre comme la simple régionalisation de l'autorité bureaucratique, mais devrait comprendre l'implication d'usagers et de leurs représentants, à tous les niveaux, dans les décisions concernant le développement et la mise en pratique des politiques (1990a, p. 2).

À cet égard, la position de la Coalition des aînés est claire :

Elles [les personnes âgées] n'accepteront qu'un partenariat égal dans le traitement de ces aspects critiques de la vie. Les aînés insistent sur leur droit de participer aux décisions concernant les politiques qui les touchent dans leur vie et dans leur bien-être, et dans la distribution de services. Ils rejettent le contrôle exercé par les professionnels et les spécialistes administratifs, mais offrent leur collaboration (1990a, p. 3).

L'AQDR $(1994$, p. 2) va dans le même sens que la Coalition lorsqu'elle constate que les organismes communautaires se sentent conviés à un "partenariat obligé », alors que c'est à un " partenariat actif » qu'ils veulent contribuer. La FADOQ propose, dès 1980, la formation d'un conseil des aînés et souhaite qu'il soit le résultat de la prise en charge des personnes âgées par elles-mêmes. Elle propose que ce conseil repose en grande partie sur le bénévolat et qu'il soit apolitique (en dehors des structures gouvernementales) pour assurer son autonomie et sa liberté d'action. De plus, elle affirme que ce conseil doit être à 
"l'affût des problèmes des déshérités 》 (1980, p. 11). En 1990, la FADOQ (1990a, 1990b) revient à la charge et demande au gouvernement du Québec de former un conseil des aînés et d'assurer « [...] une représentation des aînés au niveau des instances décisionnelles dans les domaines qui les touchent de près » (1990a, p. 4). Ce Conseil des aînés sera effectivement formé en 1993.

\section{DISCUSSION}

Cette discussion vise à déterminer dans laquelle des grandes tendances actuelles les groupes d'aînés québécois s'inscrivent. Un retour aux faits saillants émergeant de l'analyse de leurs prises de position permettra de faire le lien avec les principales caractéristiques des trois tendances définies précédemment.

\section{Un État présent et un acteur communautaire vigoureux}

D'entrée de jeu, il est possible d'établir que l'analyse des documents officiels produits par les aînés démontre leur rejet de la tendance néolibérale. Les aînés défendent les droits acquis durant la période providentialiste. Toutefois, certains éléments du discours des aînés organisés s'inscrivent dans la perspective défendue par les tenants de la troisième voie: la sociocommunautarisation. Les groupes d'aînés sont critiques par rapport à l'appareil étatique. Ils dénoncent, entre autres, la rigidité de l'appareil de l'État, la lourdeur de la bureaucratie et le contrôle directif inhérents aux services étatiques, de même que la fragmentation des interventions (AQDR, 1979; FADOQ 1986a; Forum, 1986). Cependant, contrairement aux néolibéraux, les groupes d'aînés considèrent que l'État doit jouer un rôle central dans la réponse à leurs divers besoins. Tout comme les tenants de l'État-providence, ils confèrent à l'État un rôle de maître d'œuvre et de gardien des mesures assurant la sécurité sociale des citoyens. Ils en appellent à un assainissement du système public, mais désirent conserver la gratuité, l'accessibilité et l'universalité des services gagnés dans les années providentialistes. En matière de soutien à domicile, la presque totalité des groupes d'aînés revendiquent des services en provenance du réseau public (CLSC) pour tous les aînés qui en ont besoin. Cette revendication émane aussi bien du milieu francophone que du milieu anglophone et se confirme dans le temps. Selon ces groupes, l'Etat exerce donc une fonction de fournisseur de services publics universels et gratuits. De plus, les groupes rejettent la 
solution des allocations directes suivant laquelle les bénéficiaires reçoivent une allocation afin d'engager eux-mêmes la ressource. Cette revendication de prestation en nature (en services) plutôt qu'en espèces (en argent) rejoint la conception de la sécurité sociale élaborée dans le cadre de l'État-providence (Groulx, 1990). Cependant, le rôle essentiel de l'État comme maître d'œuvre de la solidarité sociale est aussi reconnu par les tenants de la sociocommunautarisation. De plus, lorsqu'on prend connaissance de leur position à l'égard du développement des groupes communautaires et du rôle que ces groupes doivent jouer, la tendance à emprunter la voie sociocommunautaire semble se confirmer. Dès 1979, l'AQDR recommande le développement de groupes communautaires et bénévoles. La FADOQ (1986b, 1990a, 1992) est sans doute le groupe qui défend le plus ardemment la place des réseaux d'entraide et leur fonction préventive. Cependant, les groupes d'aînés, qu'ils soient anglophones ou francophones, revendiquent la reconnaissance et le soutien financier pour les groupes communautaires et bénévoles. Ils considèrent que le secteur communautaire non public peut prendre en charge certains services, en élaborant une "façon de faire " différente de celle du secteur public, plus proche des besoins des aînés. SeIon eux, la mission des groupes communautaires est une mission de prévention et de complémentarité avec celle des services du réseau public. Dans la perspective de la sociocommunautarisation, le secteur communautaire prendrait effectivement une plus grande place dans la prise en charge de la réponse aux besoins des citoyens (Groulx, 1990; Rosanvallon, 1981).

Par ailleurs, surtout depuis les années 1990, les groupes craignent une mobilisation trop forte des ressources bénévoles, qui servirait la volonté néolibérale de minimiser les interventions étatiques dans le domaine de la santé et des services sociaux. En 1995, la Coalition des aînés en appelle à l'importance de préserver l'autonomie des groupes communautaires par rapport à l'État obnubilé par son déficit budgétaire. La privatisation des services est dénoncée par l'AQDR qui se prononce, depuis 1979, très clairement contre cette solution alternative. De son côté, la FADOQ (1990c) semble laisser une place aux initiatives du privé. Les regroupements d'aînés anglophones de Montréal semblent montrer aussi moins de réticences à l'égard de la mise sur pied de services privés. Cependant, tous s'entendent pour éviter que se développent des services différents pour les riches 
et pour les pauvres. En ce sens, malgré des positions parfois divergentes, aucun groupe n'adhère à la vision néolibérale qui compte sur les lois du marché pour régulariser les rapports sociaux. Les groupes d'aînés misent avant tout sur l'intervention de l'État et sur la force du communautaire.

\section{Les valeurs de solidarité, de partage et d'égalité}

La vision des aînés sur le rôle des différents acteurs engagés dans le domaine de la santé et des services sociaux, notamment dans le soutien à domicile, repose sur des valeurs et sur une façon de voir les rapports humains qui s'apparentent à la fois à la tendance providentialiste et à la tendance sociocommunautaire. Les groupes d'aînés considèrent que l'on doit répondre collectivement à des besoins collectifs (Coalition des aînés, 1992; FADOQ, 1992). À cet effet, l'AQDR se prononce contre la privatisation des services et la Coalition des aînés (1990a) estime que les services privatisés mènent à l'inégalité, alors que les services bureaucratiques conduisent à un manque d'humanisme et à l'inflexibilité. Les groupes d'aînés s'appuient sur des principes de solidarité, de partage et d'égalité pour revendiquer l'universalité, l'accessibilité et la gratuité des services publics ainsi que le développement du secteur communautaire.

Selon la perspective sociocommunautaire, la communauté constitue le lieu le plus près de la source des problèmes et permet la coopération, l'entraide et la responsabilisation de la communauté (Beaudry et Jalbert, 1987; Groulx, 1990). L'entraide devient alors non seulement un droit, mais aussi une obligation. La FADOQ (1986b) fait justement appel à la responsabilisation des aînés : les droits appellent aussi des responsabilités. La volonté de réduire l'intervention de l'État, exprimée par les adeptes de la sociocommunautarisation, se fonde aussi sur le respect de l'autonomie des individus et des collectivités (Beaudry et Jalbert, 1987). L'autonomie est au centre des interventions des groupes d'aînés: désir de mettre en place des mesures permettant de garder ou de développer l'autonomie; revendication afin que les aînés soient reconnus dans la société comme des personnes autonomes, utiles, capables de se responsabiliser et de décider. Les aînés s'opposent cependant à ce que les organismes communautaires à caractère bénévole soient surutilisés comme pourvoyeurs de services, ce qui ne serait qu'une forme de retour à la charité publique. II s'agit là d'un reproche adressé au scénario sociocommunautaire par ses opposants désireux de conserver 
les droits sociaux déjà acquis lors de la mise en place de l'Étatprovidence.

\section{Décentralisation, citoyenneté active et coopération conflictuelle}

La vision que les groupes d'aînés ont de la démocratie et de la place des personnes âgées dans le processus décisionnel va sans équivoque dans le sens de la sociocommunautarisation. Les regroupements d'aînés insistent sur la participation active des personnes âgées elles-mêmes aux décisions les concernant. Selon eux, la décentralisation des décisions vers les instances régionales et locales favoriserait la mise sur pied de services plus près des besoins des aînés tout en leur permettant de se positionner comme membres actifs dans les différentes instances décisionnelles. Cette perspective rejoint celle du courant sociocommunautaire qui défend une forme de démocratie directe qui s'exercerait le plus près possible des citoyens (Groulx, 1990). Les groupes d'aînés voient la personne âgée comme un citoyen actif et pas seulement comme une utilisatrice de services. Là encore, ils s'inscrivent dans le scénario sociocommunautaire qui considère le bénéficiaire d'un service comme un acteur responsable socialement (Rosanvallon, 1995).

Depuis les années 1990, les aînés réclament le droit d'être traités en partenaires dans l'élaboration des politiques sociales et des programmes qui en découlent, de même que dans le développement de services communautaires (de la défense des droits aux services directs de soutien à domicile). Tout en revendiquant le soutien de l'État, ils comptent préserver leur spécificité et leur autonomie. Surtout en matière de soutien à domicile, où plusieurs groupes mettent en place des services directs d'aide à domicile, les groupes d'aînés s'engagent dans un processus de "coopération conflictuelle », présentée par Favreau et Hurtubise comme une forme de coopération « [...] au sein de laquelle la participation s'exerce certes de façon minoritaire et asymétrique mais où il est possible de se fixer des objectifs concrets que partagent d'autres acteurs tout en défendant simultanément des intérêts différents, voire même opposés » (Favreau et Hurtubise, 1993, p. 37 qui citent Lévesque et Mager, 1992, p. 19-68). La série d'actions revendicatives menées par la Coalition des aînés avec des groupes d'assistés sociaux au 
printemps 1996 concernant les modalités de l'entrée en vigueur de l'assurance-médicaments vont dans ce sens.

\section{CONCLUSION}

Les trois grandes tendances théoriques qui cohabitent au Québec (socioétatique, néolibérale, sociocommunautaire) sont présentes simultanément dans l'élaboration des politiques sociales. Les tenants de chacune de ces tendances défendent leurs positions respectives, afin d'influencer la forme de rapport qui devra s'établir entre l'État et la société civile. L'analyse de la position des groupes d'aînés québécois révèle leur opposition au néolibéralisme et leur défense de certains traits caractérisant le providentialisme traditionnel et la tendance sociocommunautaire. L'analyse des documents produits par les regroupements d'aînés est révélatrice non seulement de leur vision sur les politiques concernant la santé et les services sociaux, mais aussi de l'évolution de leur réflexion. Par exemple, le droit à des services universels, accessibles et gratuits est un cheval de bataille auquel les aînés sont demeurés fidèles depuis 1979. II en est de même de la demande de soutien pour le développement et le financement des groupes communautaires qui s'appuient sur l'entraide et le volontariat. Par contre, depuis les années 1990, les groupes d'aînés craignent qu'une perspective de désengagement de l'État ne favorise une surutilisation des services offerts par des groupes communautaires à caractère bénévole.

Cet article visait avant tout à décrire la position des groupes d'aînés québécois à partir des mémoires qu'ils ont rédigés de 1979 à 1995. II serait intéressant, lors de travaux ultérieurs, d'élargir l'analyse aux tendances de ces regroupements en ce qui a trait à certains enjeux sociaux spécifiques et aux rapports qu'ils entretiennent avec les autres groupes de la société. À cet égard, nous considérons que le matériel étudié et l'angle d'analyse privilégié dans cet article imposent des limites. Cependant, nos réflexions nous amènent à concevoir l'hypothèse de l'émergence d'un mouvement social des aînés. Leur participation de plus en plus active aux débats politiques, la formation de regroupements provinciaux et de la Coalition des aînés québécois, l'évolution dans le temps d'une action et d'un discours qui se portent à la défense des droits des aînés devant une offensive visant à les exclure de la population dite active, le développement d'une vision de leur rôle comme acteurs actifs d'un projet 
de société qui repose sur des valeurs comme l'égalité et la solidarité sont autant d'indices qui fondent cette hypothèse. Celle-ci pourrait faire l'objet de travaux ultérieurs qui devraient examiner les trois composantes d'un mouvement social, à savoir la construction d'une identité de groupe, l'opposition à un adversaire clairement identifié et la promotion d'un projet de société (Neveu, 1996). La seule lecture de l'extrait d'un document produit par la FADOQ en 1992 rend plausible l'hypothèse de l'émergence d'un mouvement social des aînés :

Aujourd'hui, à travers leurs clubs et associations, les 50 ans et plus se battent... pour les générations qui les suivent. Leur combat est essentiel puisque ce qui est perdu aujourd'hui le sera aussi demain, pour les prochaines cohortes de 50 ans et plus. Leur parole, enracinée dans le temps, nous est donc essentielle. Elle fait contrepoids et nous rappelle qu'on ne peut pas couper la vie en morceaux ni abdiquer nos responsabilités. L'une d'entre elles, c'est de faire en sorte que les personnes vieillissantes aient, dans notre société, droit de parole, de participation et de créativité, pour la suite du monde " $(1992$, p. 66).

Andrée SÉVIGNY

Travailleuse sociale et étudiante au doctorat Membre du Centre de recherche sur les services communautaires École de service social Université Laval

Yves HURTUBISE Professeur titulaire École de service social Université Laval 


\section{Références bibliographiques}

BEAUCHEMIN, J., G. BOURQUE et J. DUCHASTEL (1995). « Du providentialisme au néolibéralisme de Marsh à Axworthy. Un nouveau discours de légitimation de la régulation sociale ", Cahiers de recherche sociologique, $\mathrm{n}^{\circ} 24$, p. 15-47.

BEAUDRY, L. et L. JALBERT (1987). "Le néolibéralisme, signification et portée politiques ", dans L. Beaudry et L. Jalbert, Les métamorphoses de la pensée libérale, Sainte-Foy, Presses de I'Université du Québec, p. 9-27.

FAVREAU, L. et Y. HURTUBISE (1993). CLSC et communautés locales : la contribution de l'organisation communautaire, SainteFoy, Presses de l'Université du Québec.

GARANT, L. et M. BOLDUC (1990). L'aide par les proches: mythes et réalités, vol. 8, ministère de la Santé et des Services sociaux, Direction de l'évaluation, coll. Études et analyses.

GODBOUT, J.-T. (1995). "La troisième génération de l'Étatprovidence », dans J.-L. Klein et B. Lévesque (dir.), Contre l'exclusion: repenser l'économie, Sainte-Foy, Presses de l'Université du Québec, p. 235-245.

GROULX, L.-H. (1990). Où va le modèle suédois? État-providence et protection sociale, Paris, L'Harmattan et Montréal, Presses de l'Université de Montréal.

GUBERMAN, N. et P. MAHEU (1994). "Au-delà des soins: un travail de conciliation », Service social, vol. 43, n 1, p. 87-104.

LAMOUREUX, J. (1994). Le partenariat à l'épreuve, Montréal, Les Éditions Saint-Martin.

MAYER, R. (1994). "L'évolution des services sociaux ", dans F. Dumont, S. Langlois et Y. Martin (dir.), Traité des problèmes sociaux, Québec, Institut québécois de recherche sur la culture, p. 1013-1033.

MINISTĖRE DE LA SANTÉ ET DES SERVICES SOCIAUX (1988). Rapport de la Commission d'enquête sur les services de santé et les services sociaux (commission Rochon), Québec, Gouvernement du Québec.

NEVEU, E. (1996). Sociologie des mouvements sociaux, Paris, La Découverte.

PLAMONDON, D. (1991). "Vers un modèle de services sociosanitaires alternatifs », Intervention, $\mathrm{n}^{\circ} 90$, p. 72-77.

PRESSE CANADIENNE (1996). "Assurance-médicaments : assez, c'est assez, disent les aînés ", Le Devoir, 29-05, p. A-8.

ROSANVALLON, P. (1981). La crise de l'État-providence, Paris, Seuil. 
ROSANVALLON, P. (1995). La nouvelle question sociale, Paris, Seuil. SORMAN, Guy (1984). La solution libérale, Paris, Fayard.

VAILLANCOURT, Y. et C. JETTÉ (1997). Vers un nouveau partage des responsabilités dans les services de santé : rôles de l'État, du marché, de l'économie sociale et du secteur informel, Montréal, Cahiers du LAREPPS, Département de travail social, Université du Québec à Montréal. 